\title{
Exendin-4 Plays a Protective Role in a Rat Model of Spinal Cord Injury Through SERCA2
}

\author{
Zhonglei Sun ${ }^{a, b}$ Yingfu Liu ${ }^{c}$ Xianbin Konga,e Renjie Wang ${ }^{a} \quad$ Yunqiang $X^{d}{ }^{d}$

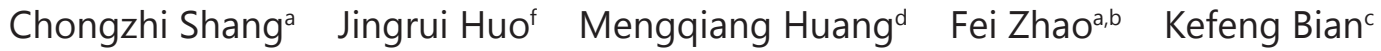 \\ Sai Zhang ${ }^{\text {a }}$ Yue Tu $^{\text {a }}$ Xuyi Chen ${ }^{\mathrm{a}}$ \\ aTianjin Key Laboratory of Neurotrauma Repair, Institute of Traumatic Brain Injury and Neuroscience, \\ Center for Neurology and Neurosurgery, Affiliated Hospital of the Logistics University of the Chinese \\ People's Armed Police Force, Tianjin, bJinzhou Medical University, Jinzhou, 'Logistics University \\ of People's Armed Police Force, Tianjin, ${ }^{\mathrm{d}}$ Tianjin Medical University, Tianjin, ${ }^{\mathrm{e}}$ Tianjin University of \\ Traditional Chinese Medicine, Tianjin, ${ }^{f}$ Cangzhou Medical College, Hebei, China
}

\section{Key Words}

Spinal cord injury(SCI) $\bullet$ SERCA2 - Exendin-4 • Apoptosis

\begin{abstract}
Background/Aims: Current therapies for spinal cord injury (SCI) have limited efficacy, and identifying a therapeutic target is a pressing need. Sarcoplasmic/endoplasmic reticulum $\mathrm{Ca}^{2+}$ ATPase 2 (SERCA2) plays an important role in regulating calcium homeostasis, which has been shown to inhibit apoptosis. Exendin-4 has been shown to inhibit the apoptosis of nerve cells in $\mathrm{SCI}$, which can also improve SERCA2 expression. In this study, we sought to determine whether exendin-4 plays a protective role in a rat model of SCI via SERCA2. Methods: To investigate the effects of exendin-4 on SCI, a rat model of SCI was induced by a modified version of Allen's method. Spinal cord tissue sections from rats and western blot analysis were used to examine SERCA2 expression after treatment with the long-acting glucagon-like peptide 1 receptor exendin-4 or the SERCA2 antagonist 5(6)-carboxyfluorescein diacetate $\mathrm{N}$-succinimidyl ester (CE). Locomotor function was evaluated using the Basso Beattie Bresnahan locomotor rating scale and slanting board test. Results: Cell apoptosis was increased with CE treatment and decreased with exendin-4 treatment. Upregulation of SERCA2 in female rats with SCI resulted in an improvement of motor function scores and histological changes. Conclusion: These findings suggest that exendin-4 plays a protective role in a rat model of SCI through SERCA2 via inhibition of apoptosis. Existing drugs targeting SERCA2 may be an effective therapeutic strategy for the treatment of SCI.

Sun ZL, Liu YF and Kong XB contributed equally to this paper.

Xuyi Chen, MD, PhD and

Yue Tu, MD, PhD

KARGER
Tianjin Key Lab. of Neurotrauma Repair, Inst. of Traumatic Brain Injury and Neuroscience, Center for Neurology and Neurosurgery; Affiliated Hospital of the Logistics Univ. of the Chinese People's Armed Police Force, Tianjin 300162, (China); Tel. +86-18920677110, E-Mail chenxuyi1979@sina.com or ytumail@vip.126.com 


\section{Introduction}

Spinal cord injury (SCI) has a high incidence among young patients and is one of the most common diseases in the world with high morbidity, high disability, and high treatment costs [1-3]. Direct or indirect trauma leads to the following pathological phases of SCI: primary mechanical injury and secondary injury, which lead to complex cellular and molecular changes at the injury site of the central nervous system, as well as loss of nerve connections that result in functional behavioral defects [4]. Therefore, due to the inability to generate new neurons in the spinal cords of adults, choosing a suitable target to inhibit the apoptosis of nerve cells can effectively reduce the irreversible loss of neurons in SCI and improve nerve function.

Although the molecular mechanism of SCI pathogenesis remains poorly understood, there is evidence that SCI-induced ion imbalances and excess production of reactive oxygen free radicals and subsequent apoptotic necrosis are critical for secondary SCI damage [5]. The dynamic balance of ions, especially the disturbance of calcium homeostasis, has attracted attention in many forms of pathological axonal injury and as a major cause of neuronal dysfunction [6-8]. Calcium passes through a voltage-gated calcium channel and is isolated and buffered to ensure that a steady state of calcium is maintained. Calcium extrusion from neurons is mediated by plasma membrane calcium ATPase and sodium-calcium exchange, whereas the endoplasmic reticulum utilizes sarcoplasmic/endoplasmic reticulum $\mathrm{Ca}^{2+}$ ATPase (SERCA) [9]. Any of these processes can increase intracellular calcium to abnormally high levels, which can lead to damage, including the release of mitochondrial cytochrome $\mathrm{C}$ and activation of apoptotic caspase cascades [10]. Moreover, studies have shown that the effective activation of SERCA2 can inhibit endoplasmic reticulum oxidative stress, block apoptosis, and provide appropriate organ protection $[11,12]$. However, the mechanisms underlying the SERCA2-associated development of SCI are not clear.

Exendin-4, which is a long-acting glucagon-like peptide 1 receptor agonist, has been shown to be valuable in the study of SERCA2. Studies have shown that exendin-4 can promote the expression of SERCA2 [13]. Exendin-4 can also reverse insulin resistance in macrophages by antagonizing endoplasmic reticulum stress and apoptosis by promoting the reversal of SERCA2 expression [14]. The present study was designed to investigate the role of SERCA2 upregulation in SCI and elucidate the possible mechanisms. We found that knockdown of SERCA2 increased cell apoptosis, but promotion of SERCA2 expression with exendin-4 inhibited cell apoptosis, which resulted in improved recovery of SCI. We suggest that exendin-4 plays an important role in SCI through SERCA2 via inhibiting apoptosis, and this conclusion provides a new approach for SCI research.

\section{Materials and Methods}

\section{Animals}

Female adult Sprague-Dawley rats weighing 200-220 g were provided by the Experimental Animal Center of the Academy of Military Medical Sciences (license no. SCXK-[Military2012-0004]) for the experiments. All rats were maintained on a 12-h light-dark cycle with free access to food and water. All experimental procedures and protocols were approved by the Institutional Animal Care and Use Committee of the Affiliated Hospital of the Logistics University of the Chinese People's Armed Police Force, China. All efforts were made to minimize the number of animals used and their suffering.

\section{Materials}

5(6)-Carboxyfluorescein diacetate N-succinimidyl ester (CE; SERCA2 inhibitor), RPMI 1640, fetal bovine serum, horse serum, L-glutamine, penicillin G, and streptomycin were purchased from SigmaAldrich (St. Louis, MO, USA). PKA inhibitor (PKI) (6-22) amide, Rp-cyclic AMPS (cAMP inhibitor), and exendin-4 were purchased from Cayman Chemical Company (Ann Arbor, MI, USA). Primary antibodies for SCI rat spinal cord tissue included PKA (rabbit, ab75991; Abcam, Cambridge, MA, USA), SERCA2 


\section{Cellular Physiology Cell Physiol Biochem 2018;47:617-629 \begin{tabular}{l|l|l} 
and Biochemistry $10.1159 / 000490017$ & $\begin{array}{l}\text { DO } 2018 \text { The Author(s). Published by S. Karger AG, Basel } \\
\text { Published online: May 25, } 2018\end{array}$ \\
\hline
\end{tabular}}

Sun et al.: Exendin-4 Protect Spinal Cord Injury by SERCA2

(mouse, ab2817; Abcam), caspase-3 (mouse, ab13585; Abcam), beclin-1 (rabbit, ab207612; Abcam), Bcl2 (rabbit, ab32124; Abcam), Bax (rabbit, ab32503; Abcam), and $\beta$-tubulin (rabbit, ab1083424; Abcam). For immunofluorescence analysis, secondary antibodies were purchased from Proteintech and used for western blotting. Other reagents included an enhanced chemiluminescence system (ECL kit; Beyotime Institute of Biotechnology, Nanjing, China), terminal deoxynucleotidyl transferase dUTP nick-end labeling kit (TUNEL kit; Roche, Basel, Switzerland), cAMP kit (R\&D Systems, Minneapolis, MN, USA), normal donkey sera (Jackson, ImmunoResearch, Gruber, PA, USA), 4',6-diamidino-2-phenylindole (DAPI; Cayman Chemical, Ann Arbor, MI, USA), BCA protein assay kit (Beyotime Institute of Biotechnology), polyvinylidene difluoride membranes (SEQ00010, $13.5 \times 15 \mathrm{~cm}$; Millipore, Bedford, MA, USA), and bovine serum albumin (BSA; AMRESCO, Solon, OH, USA). The equipment included a light microscope (IX71; Olympus, Tokyo, Japan) and an inverted fluorescence microscope (Eclipse T3-S; Nikon, Tokyo, Japan). PC12 cells were acquired from the Riken Cell Bank (Tsukuba Science City, Japan).

\section{Experimental design}

In vitro experiments

To determine the effects of exendin- 4 on SERCA2 expression, PC12 cells were divided into 4 groups: sham group (no treatment), Exendin-4 group (treated with $100 \mathrm{nM}$ exendin-4 for $6 \mathrm{~h}$ ), PKI group (treated with $0.5 \mu \mathrm{M}$ PKI [6-22] amide for $12 \mathrm{~h}$ ) [15], and PKI+exendin-4 group (treated with $0.5 \mu \mathrm{M} / \mathrm{L}$ PKI [6-22] amide for $12 \mathrm{~h}$, and then treated with $100 \mathrm{nM}$ exendin-4 for $6 \mathrm{~h}$ ) [16]. After incubation for an appropriate period, cells in 24-well plates were washed twice with cold phosphate-buffered saline (PBS), lysed by adding $40 \mu \mathrm{L}$ sodium dodecyl sulfate (SDS) sample buffer (62.5 mM Tris-HCl [pH 6.8], 2\% SDS, 10\% glycerol, 50 $\mathrm{mM}$ dithiothreitol, $0.1 \%$ bromophenol blue), and scraped. Lysates were boiled for $10 \mathrm{~min}$ and centrifuged. Proteins in the extracts were examined by western blot analysis.

\section{In vivo experiments}

The main purpose of this study was to explore the potential of SERCA2 to modulate apoptosis and subsequently reduce SCI. The following experimental studies were performed: (i) in vivo analysis of the relationship between SERCA2 and apoptosis in SCI rats, where SERCA2 expression was determined by immunofluorescence and western blotting analyses; (ii) the upstream signaling pathway was assessed in rat spinal cord tissue by measuring cAMP/PKA and SERCA2 generation; and (iii) in vivo studies to determine the efficacy of exendin-4 to work against SCI were conducted in a rat model of SCI. The exact numbers of animals used in each experiment are indicated below and in the figure legends.

Experiment 1: Forty-eight rats were randomly divided into 4 groups ( $\mathrm{n}=12$ per group): SCI 12-h and 24-h groups (intrathecal injection with an equal volume of normal saline after SCI was induced) and CE 12-h and 24-h groups (2.7 mg/rat intrathecal injection of CE after SCI was induced) [17]. Each group was randomly divided into 2 subgroups ( $\mathrm{n}=6$ per group) for the following experiments: (A) tissue sections and (B) western blot analysis. After each time point, the rats were sacrificed with an overdose of chloral hydrate and transcardially perfused with ice-cold PBS. The spinal cords (T9-11) were removed immediately to identify medulla spinal pathological changes, examine SERCA2 expression, and observe apoptosis using a western blot assay, immunofluorescence assay, and TUNEL assay, respectively.

Experiment 2: Eighteen rats were randomly divided into 3 groups ( $=6$ per group): sham group (intrathecal injection with normal saline), PKI group (intrathecal injection of $10 \mu \mathrm{g} / \mathrm{kg}$ PKI [6-22] amide) [18], and Rp-cAMP group (intrathecal injection of $200 \mu \mathrm{g} / \mathrm{rat} \mathrm{Rp}$-cyclic AMPS) [19, 20]. The rats were sacrificed at $24 \mathrm{~h}$ after surgery, and the spinal cords (T9-11) were removed immediately to identify cAMP/ PKA and SERCA2 expression by western blot analysis.

Experiment 3: To select the appropriate exendin-4 dose, 42 rats were randomly divided into 7 groups. After SCI surgery, the rats were given intraperitoneal injections of $0.1,0.5,1,2,5,10$, or $25 \mu$ g exendin-4/ rat/day. After 3 days, the rats were euthanized, and spinal cord tissue was immediately collected. The relative content of SERCA2 was detected by western blot analysis, then the appropriate exendin-4 dose was selected by goodness of fit analysis. Next, 72 rats were randomly divided into 4 groups ( $\mathrm{n}=18$ per group): sham group (laminectomy only), SCI group (SCI was induced followed by an intraperitoneal injection of saline with the same volume as the drug groups immediately after injury), and 2 and $10 \mu$ g exendin-4 groups (SCI was induced followed by an intraperitoneal injection of 2 or $10 \mu \mathrm{g}$ exendin-4/rat in saline immediately after surgery). Each group was divided randomly into 3 subgroups ( $n=6$ per group) for the following 


\section{Cellular Physiology Cell Physiol Biochem 2018;47:617-629 \begin{tabular}{c|c|c|} 
DOI: 10.1159/000490017 & $\begin{array}{l}\text { O } 2018 \text { The Author(s). Published by S. Karger AG, Basel } \\
\text { wwww.karger.com/cpb }\end{array}$
\end{tabular}}

Sun et al.: Exendin-4 Protect Spinal Cord Injury by SERCA2

experiments: (A) behavioral analysis of motor function, (B) western blot analysis, and (C) tissue sections. The daily subcutaneous injections of saline or exendin- 4 were performed at similar times of the day for each rat. The rats were sacrificed at the end of the experiment (at 3 days and 4 weeks after surgery), and the spinal cords (T9-11) were removed immediately to identify medulla spinal pathological changes, examine the expression of SERCA2 and its upstream proteins, and observe apoptosis by hematoxylin-eosin staining, western blot analysis, and TUNEL assays, respectively.

\section{PC12 cell culture}

PC12 cells were placed in RPMI 1640 medium supplemented with 5\% fetal bovine serum, 10\% horse serum, $2 \mathrm{mM} \mathrm{L}$-glutamine, $100 \mathrm{U} / \mathrm{mL}$ penicillin $\mathrm{G}$, and $100 \mathrm{mg} / \mathrm{mL}$ streptomycin at $37^{\circ} \mathrm{C}$ in a humidified atmosphere of $5 \% \mathrm{CO}_{2}$ and $95 \%$ air, as described previously [21].

\section{SCI model}

The rat model of SCI was induced using an improved impactor based on Allen's method [9, 22]. The rats were anesthetized and injected intraperitoneally with chloral hydrate $(300 \mathrm{mg} / \mathrm{kg})$. The incision area was sterilized with $75 \%$ alcohol, an incision was made, and the skin was separated, then a laminectomy was performed to expose spinal cord segment T10. The impactor (weighing $10 \mathrm{~g}, 3 \mathrm{~mm}$ in diameter, and 200 $\mathrm{mm}$ in length) was obtained from the Affiliated Hospital of the Logistics University of the Chinese People's Armed Police Force. The impactor was dropped from a height of $50 \mathrm{~mm}$ to the surface of the spinal cord. Successfully induced SCI resulted in spinal cord congestion, tail swing reflexes, swaying legs, and slow paralysis. The wound was sutured after the spinal cord was hit. All rats were kept in a separate environment at $24^{\circ} \mathrm{C}$ to ensure adequate water, food, and clean bedding. The rats were provided intermittently with assisted urination 3 times daily.

\section{Behavioral tests}

Hindlimb motor function assessments were performed at 1, 3, 7, 14, and 28 days after SCI using the Basso Beattie Bresnahan (BBB) locomotor rating scale [23] and slanting board test [24]. The BBB score ranged from 0 (complete hindlimb paralysis) to 21 (normal locomotion). The score was determined by the motor capacity of the experimental rats, including the movement of the hindlimbs, weight-bearing, and coordination of forelimb and hindlimb movements. The rats were placed in an open field of wooden models for 4 min and scored by 2 researchers using blinded methods.

The slanting board test with the Rivlin method involved placing a rat on a rectangular wooden oblique board covered with a rubber pad, and the oblique plate was rotated to measure the angle of the inclined plate. When the longitudinal axis of the rat was parallel to the longitudinal axis of the inclined plate, the rat was tilted toward the side of the tilted plate to raise it by 5 increments. The maximum angle at which a rat could maintain its position for $5 \mathrm{~s}$ was measured. Each animal was measured 5 times, and the average was taken as the measured value.

\section{Detection of cAMP concentration}

To detect the content of cAMP in the spinal cord, fresh spinal cord tissue was added to PBS on ice at a ratio of 1:10 to grind it into a homogenate, which was stored at $-80^{\circ} \mathrm{C}$. The cAMP content of the spinal cord homogenates was measured by a cAMP parameter assay kit (R\&D Systems) following the standard protocol.

\section{Immunofluorescence assay}

Sections were washed in PBS containing 0.1\% Triton X-100 (PBST) for 30 min. Non-specific antibodies were blocked with 5\% BSA and 10\% normal donkey sera for $60 \mathrm{~min}$. SERCA2/DAPI fluorescent labeling staining was performed. The sections were first incubated with a rabbit primary antibody against SERCA2 (1:500) for $24 \mathrm{~h}$ at $4^{\circ} \mathrm{C}$ overnight. The sections were subsequently rinsed with PBS and incubated with a secondary antibody (donkey anti-rabbit IgG conjugated to a red fluorophore; 1:1000) for $1 \mathrm{~h}$ at room temperature. Finally, the sections were mounted with DAPI. All sections were observed with light microscopy at high magnification (scale $=20 \mu \mathrm{m}$ ). Quantification was conducted by calculating the number of positive cells in each of 10 randomly selected fields. Three segments were selected from the center of each animal (approximate head-tail length of $250 \mathrm{~mm}$ ). Each group examined contained 6 rats. The total number of labeled positive cells was analyzed.

\section{KARGER}




\section{Cellular Physiology Cell Physiol Biochem 2018;47:617-629 and Biochemistry Published online: May 25, $2018 \quad \begin{aligned} & \text { DOI: 10.1159/000490017 } 2018 \text { The Author(s). Published by S. Karger AG, Basel } \\ & \text { www.karger.com/cpb }\end{aligned}$}

Sun et al.: Exendin-4 Protect Spinal Cord Injury by SERCA2

Western blot analysis

After the experimental rats were euthanized, western blotting analysis was conducted. Protein concentration was evaluated with a BCA protein assay kit. Proteins were separated using SDS-polyacrylamide gel electrophoresis and transferred to a polyvinylidene difluoride membrane. The membrane was blocked with $0.1 \%$ BSA in PBS for $1 \mathrm{~h}$ at room temperature and then incubated with the following primary antibodies in PBST overnight at $4^{\circ} \mathrm{C}$ : rabbit anti-PKA (1:1000), mouse anti-SERCA2 (1:1000), mouse anti-caspase-3 (1:1000), rabbit anti-beclin-1 (1:1000), rabbit anti-Bcl-2 (1:1000), rabbit anti-Bax (1:1000), or rabbit anti$\beta$-tubulin (1:1000). The membrane was incubated with a goat anti-rabbit secondary antibody (1:2000) for $1 \mathrm{~h}$ at room temperature. Immunoreactive bands were detected using an enhanced chemiluminescence system (ECL kit), and the images were analyzed with ImageJ 1.42q software.

\section{Hematoxylin-eosin staining}

The first segment of the spinal cord $\left(\mathrm{L}_{9-11}\right)$ was dissected and post-fixed in $4 \%$ paraformaldehyde for $48 \mathrm{~h}$. After dehydration, the spinal cords were embedded in paraffin and 5- $\mu$ m-thick serial longitudinal sections were collected. To observe histopathological changes, the sections were subjected to hematoxylineosin staining. Sections ( $5-\mu$ m-thick) were stained with hematoxylin solution for 8 min followed by 5 dips in $1 \%$ acid ethanol $(1 \% \mathrm{HCl}$ in $70 \%$ ethanol), and the sections were rinsed in distilled water. Afterwards, the sections were stained with an eosin solution for $3 \mathrm{~min}$, followed by dehydration in an alcohol gradient and cleared in xylene. An independent observer, who was blinded to the experimental design, collected images using an inverted fluorescence microscope.

TUNEL staining

Spinal cord $\left(\mathrm{L}_{9-11}\right)$ segments were subjected to antigen retrieval with proteinase $\mathrm{K}$ (Roche) for 30 min and then washed 3 times with PBS. TUNEL solution preparation and staining were performed according to the assay kit's instruction manual. Cell quantification was performed by 2 observers using an inverted fluorescence microscope at $400 \times$ magnification. Cell apoptosis (\%) was equal to the number of positive cells $/$ (positive cells + negative cells) $\times 100 \%$.

\section{Statistical analysis}

The data are presented as the mean \pm standard deviation (SD) and were analyzed using one-way analysis of variance and Tukey's post hoc multiple comparison tests. The appropriate exendin- 4 dose was selected using goodness-of-fit analysis. All experimental data were analyzed using SPSS 17.0 (Affiliated Hospital of the Logistics University of the Chinese People's Armed Police Forces). Values were considered statistically significant when $P$-values were $<0.05$.

\section{Results}

Blocking PKA activation in exendin-4-treated cells also blocks the increase in SERCA2 levels

To determine the effects of exendin-4 on SERCA2 expression, PC12 cells were inhibited with the PKA inhibitor PKI (6-22) amide. Inhibition of PKA $(P<0.01)$ abolished PKA and PKA-induced SERCA2 activity $(P<0.01)$ (Fig. 1A-C). Compared with the sham group, the PKI and PKI+exendin-4 groups showed significantly decreased PKA and SERCA2 expression $(P$ $<0.01)$. However, PKA and SERCA2 expression was significantly increased in the exendin-4 group $(P<0.01)$. Therefore, blocking PKA activation in exendin-4-treated cells also blocks the increase in SERCA2 levels.

\section{SERCA2 inhibition increases cell apoptosis}

To investigate the relationship between SERCA2 and apoptosis in the spinal cord, we used CE intrathecal injections to block SERCA2 in the spinal cord of SCI rats. The effect of SERCA2 on apoptosis was determined by measuring the expression levels of caspase-3, beclin-1, Bcl2 , and Bax in spinal cord tissue. Compared with the SCI groups, the CE groups had significantly decreased SERCA2 and Bcl-2 expression $(P<0.01)$, but caspase-3 and beclin-1 expression 
was significantly increased $(P<$ 0.01) (Fig. 2A-E). However, there was no significant difference between the 12-h and 24-h groups.

We examined the contribution of SERCA2 to the apoptosis response after a severe transverse crush SCI across the entire spinal cord. Full crush SCI was performed in adult rats at T10 (Fig. 2F). Tissue was collected at 12 and $24 \mathrm{~h}$ after injury and was evaluated quantitatively in vertical tissue sections at the T10 level (Fig. $2 \mathrm{G}$ ).

Im mun ofluore scence staining of SERCA2 and TUNEL was performed to identify spinal cord cell viability further. A high magnification indicated that SERCA2/DAPI-positive cells exhibited red punctate SERCA2 dots in the cytoplasm with a blue nucleus. A high magnification indicated that TUNEL/DAPIpositive cells exhibited green punctate TUNEL dots in the nucleus with a blue nucleus. The ratio of these positive cells was subsequently determined. The ratio of SERCA2-positive cells was lower in the CE groups than in the SCI groups $(P<0.01)$ (Fig. $2 \mathrm{H} \& \mathrm{I})$. Furthermore, the ratio of TUNEL-positive cells was significantly higher in the CE groups than in the $\mathrm{SCI}$ groups $(P$ $<0.01$ ) (Fig. 2J \& K). There was also no significant difference between the 12-h and 24-h groups.

The cAMP/PKA axis induces SERCA2 expression

The upstream activation of the signaling pathway in the spinal cord was assessed to measure SERCA2 activity. Inhibition of PKA $(P<0.01)$ with PKI $(6-22)$ amide abolished PKAinduced SERCA2 activity $(P<0.001)$ (Fig. 3B-D). In addition, compared with sham, inhibition of cAMP with Rp-cyclic AMPS reduced cAMP (Fig. 3A), PKA, and SERCA2 production $(P<$ 0.01) (Fig. 3B-D).

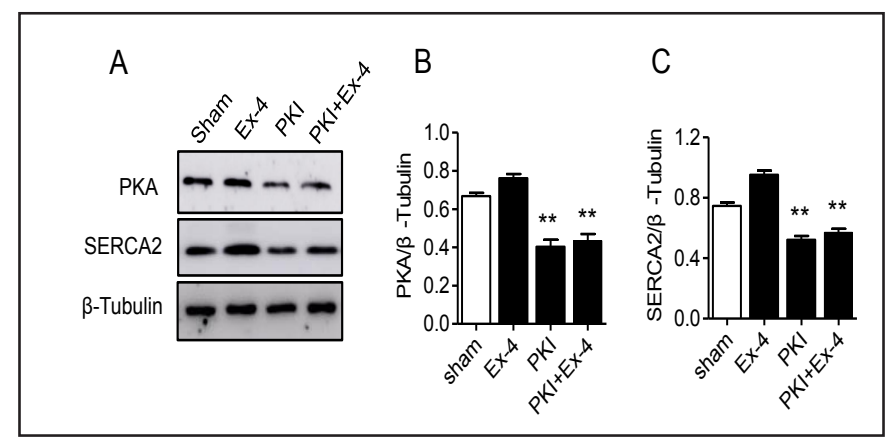

Fig. 1. Blocking PKA activation in exendin-4-treated cells also blocks the increase in SERCA2 levels. (A-C) Western blot analysis of PKA, SERCA2, and $\beta$-tubulin. Values are expressed as the mean $\pm \mathrm{SD} .{ }^{* *} \mathrm{P}<0.01$.

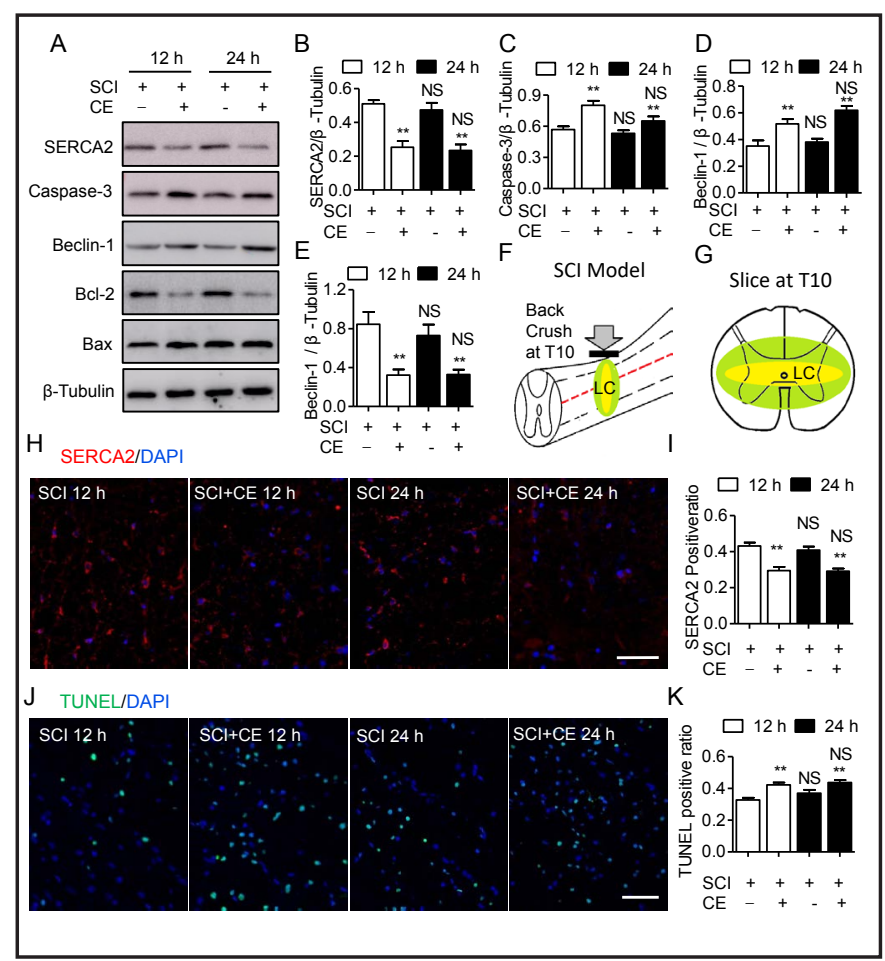

Fig. 2. Inhibition of SERCA2 increases cell apoptosis. (A-E) Western blot analysis of SERCA2, caspase-3, beclin-1, Bcl-2, Bax, and $\beta$-tubulin. (F\&G) Schematic of the SCI lesion area. (H) Immunofluorescence assessment of SERCA2. Scale bar $=50 \mu \mathrm{m}$. (I) Proportion of SERCA2-positive cells. (J) Immunofluorescence assessment of TUNEL. Scale bar $=100 \mu \mathrm{m}$. $(\mathrm{K})$ Proportion of TUNELpositive cells. Values are expressed as the mean $\pm \mathrm{SD}$. ${ }^{* *} \mathrm{P}<0.001$, compared with the SCI group. NS, not significant; LC, lesion core. 
Immunofluorescence staining of SERCA2 was performed to identify spinal cord viability further. A high magnification indicated that SERCA2/DAPI-positive cells showed red punctate SERCA2 dots in the cytoplasm with a blue nucleus. A high magnification indicated that TUNEL/DAPI-positive cells presented with green punctate TUNEL dots in the nucleus with a blue nucleus. Next, the proportion of these positive cells was quantified. The ratio of SERCA2-positive cells was lower in the PKI (6-22) amide and Rp-cyclic AMPS groups than in the sham group $(P<$ 0.01) (Fig. 3E \& F).

\section{Exendin-4 treatment promotes SERCA2 expression via the cAMP/ PKA signaling pathway \\ To select the appropriate} dose of exendin-4, SERCA2 protein was determined in spinal cord tissues from the 7 groups by western blot analysis (Fig. 4A). According to the expression level of SERCA2 protein, we chose goodnessof-fit analysis to determine the optimal dose of exendin-4. The concentration for $50 \%$ of the maximal effect (EC50) was $2.15 \mu \mathrm{g}$ exendin-4/rat. At the same time, a dose of $10 \mu \mathrm{g}$ exendin-4/rat was the most common concentration for a 100\% maximal effect (Fig. 4B). This finding demonstrates that $2 \mu \mathrm{g} / \mathrm{rat}$ and $10 \mu \mathrm{g} /$ rat doses of exendin- 4 were the most effective in our experiments.

The effect of exendin- 4 on SERCA2 was determined by measuring the expression of SERCA2 and its upstream protein cAMP/PKA in the spinal cord. Compared with the sham group, the SCI group showed significantly reduced cAMP/PKA and SERCA2 expression $(P<0.01)$ (Fig. 4C-F). However, cAMP/PKA and SERCA2 levels were increased in both exendin-4-treated groups,

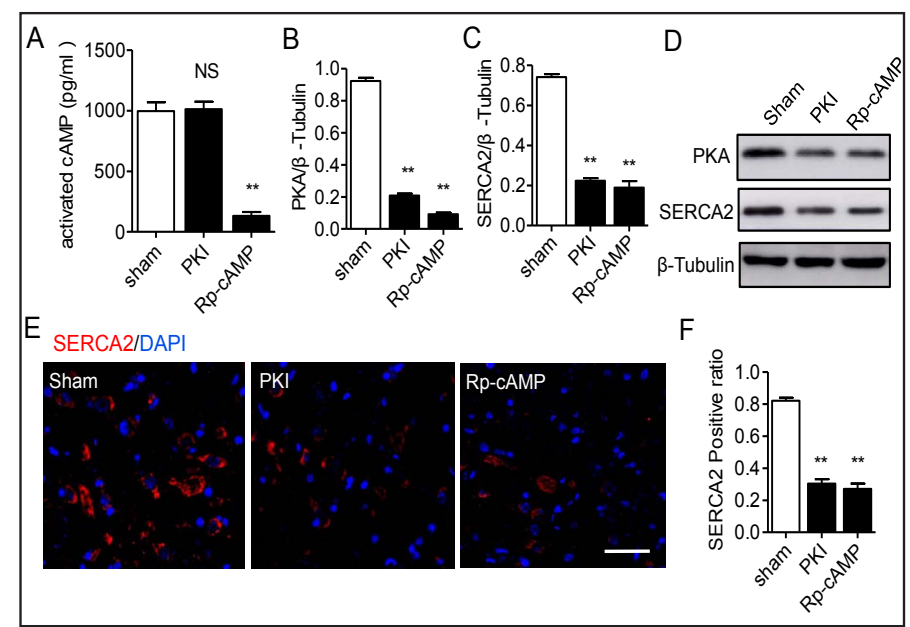

Fig. 3. cAMP/PKA pathway contributes to SERCA2 expression. (A) cAMP expression inhibits cAMP and PKA. (B-D) Western blot assessment of PKA and SERCA2 following inhibition of cAMP and PKA. (E) Immunofluorescence assessment of SERCA2. Scale bar $=200 \mu \mathrm{m}$. (F) Proportion of SERCA2-positive cells. Values are expressed as the mean $\pm \mathrm{SD} .{ }^{* *} \mathrm{P}<0.01$, compared with the sham group. NS, not significant, compared with the sham group.

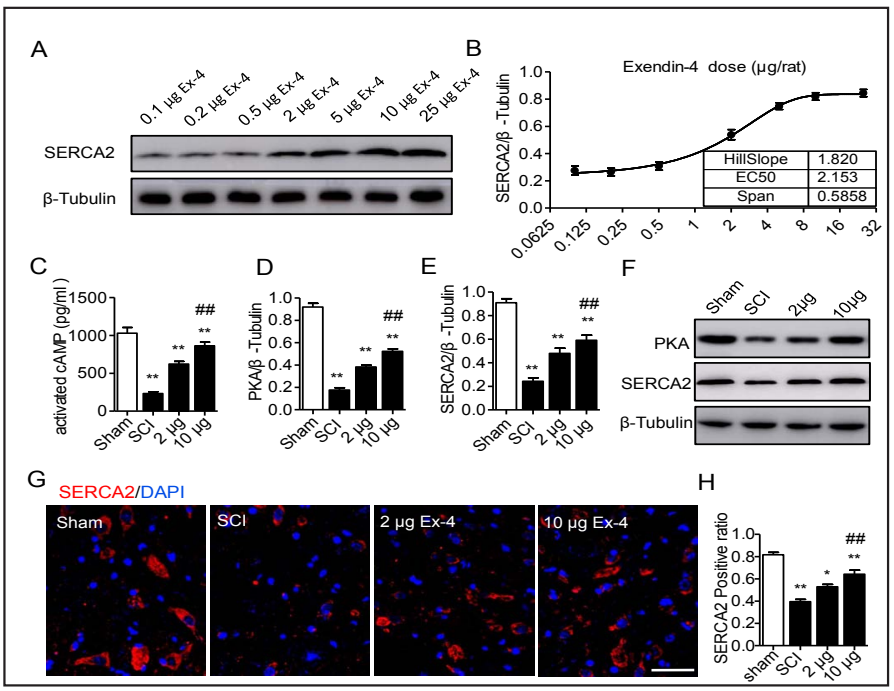

Fig. 4. Exendin-4 treatment promotes SERCA2 expression. (A-B) Dose response of the effect of exendin-4 on SERCA2 expression after the abdominal administration of exendin-4 $(0.1 \mu \mathrm{g} / \mathrm{rat} ; 0.2 \mu \mathrm{g} /$ rat; $0.5 \mu \mathrm{g} / \mathrm{rat} ; 2 \mu \mathrm{g} / \mathrm{rat} ; 5 \mu \mathrm{g} / \mathrm{rat} ; 10 \mu \mathrm{g} / \mathrm{rat}$; and $25 \mu \mathrm{g} / \mathrm{rat}$, all $\mathrm{n}=$ 6). (C) ELISA assessment of cAMP. (D-F) Western blot analysis of PKA, SERCA2, and $\beta$-tubulin. (G) Immunofluorescence assessment of SERCA2. Scale bar $=50 \mu \mathrm{m}$. $(\mathrm{H})$ Proportion of SERCA2-positive cells. Values are expressed as the mean $\pm \mathrm{SD}$. ${ }^{*} \mathrm{P}<0.05,{ }^{* *} \mathrm{P}<0.01$, ${ }^{* * *} \mathrm{P}<0.001$, compared with the sham group; ${ }^{\# \#} \mathrm{P}<0.01$, compared with the SCI group. 


\section{Cellular Physiology Cell Physiol Biochem 2018;47:617-629 and Biochemistry \begin{tabular}{l|l} 
DOI: 10.1159/000490017 & $\begin{array}{l}\text { C } 2018 \text { The Author(s). Published by S. Karger AG, Basel } \\
\text { www.karger.com/cpb }\end{array}$
\end{tabular} \\ Sun et al.: Exendin-4 Protect Spinal Cord Injury by SERCA2}

which indicates that exendin-4 improved SERCA2 expression in the spinal cord $(P<0.01)$. Furthermore, the protein levels of cAMP, PKA, and SERCA2 were significantly higher in the $10 \mu$ g exendin-4 group than in the $2 \mu$ g exendin-4 group $(P<0.01)$ (Fig. 4C-F).

Immunofluorescence staining of SERCA2 was performed to identify spinal cord cell viability further. A high magnification indicated that SERCA2/DAPI-positive cells exhibited red punctate SERCA2 dots in the cytoplasm with a blue nucleus. The ratio of these positive cells was subsequently counted. The ratio of SERCA2-positive neurons was higher in both exendin-4 groups than in the SCI group $(P<0.01)$ (Fig. 4G \& H). Furthermore, the ratio of SERCA2-positive neurons was significantly higher in the $10 \mu \mathrm{g}$ exendin-4 group than in the 2 $\mu \mathrm{g}$ exendin-4 group $(P<0.05)$ (Fig. 4G \& H).

\section{Exendin-4 inhibits apoptosis in the spinal cord}

To investigate the effects of exendin- 4 on the inhibition of apoptosis after SCI, caspase-3, beclin-1, Bcl-2, and Bax were analyzed using western blotting (Fig. 5A). Compared with the sham group, the SCI group showed significantly increased caspase- 3 and beclin-1 expression $(P<0.01)$ (Fig. 5B \& C), but significantly decreased Bcl-2 expression $(P<0.01)$ (Fig. 5D), suggesting that apoptosis was induced by SCI. However, the level of caspase-3 was decreased in both exendin-4-treated groups, but Bcl-2 and Beclin-1 levels were increased in both exendin-4-treated groups, which indicates that exendin-4 inhibited apoptosis $(P<0.01)$ (Fig. 5B \& D). Furthermore, the level of caspase- 3 was significantly lower in the $10 \mu \mathrm{g}$ exendin- 4 group than in the $2 \mu \mathrm{g}$ exendin-4 group $(P<0.01$ ) (Fig. 5B), but the Bcl- 2 and beclin-1 levels were significantly higher in the $10 \mu$ g exendin-4 group than in the $2 \mu$ g exendin-4 group $(P<$ 0.05 ) (Fig. 5C \& D), which demonstrates that the higher dose of exendin-4 was more effective for the inhibition of apoptosis.

Immunofluorescence TUNEL staining was performed to identify apoptosis further. A high magnification indicated that TUNEL/DAPI-positive cells exhibited green punctate TUNEL dots in the nucleus with a blue nucleus. The positive cell ratio was subsequently measured. The number of TUNEL-positive cells was lower in both exendin-4 groups than in the SCI group $(P<0.01)$ (Fig. 5E \& F). Furthermore, the ratio of TUNEL-positive cells was significantly lower in the $10 \mu \mathrm{g}$ exendin- 4 group than in the $2 \mu \mathrm{g}$ exendin-4 group $(P<$ 0.05 ) (Fig. 5E \& F). These findings provide biochemical and immunofluorescent evidence that autophagy was promoted by exendin- 4 after SCI, especially in the high-dose group.

\footnotetext{
Exendin-4 affects histological changes associated with SCI

Hem a toxylin-e os in staining demonstrated well-maintained spinal cord tissues in the sham group, and the neurons were morphologically normal with clear karyosomes, uniformly stained cytoplasm, and few vacuolar changes. At 3 days after surgery, spinal cord tissue defects were seen clearly in the SCI group, and the number of normal neurons was significantly decreased. The neurons were swollen and pyknotic, and the cytoplasm was stained

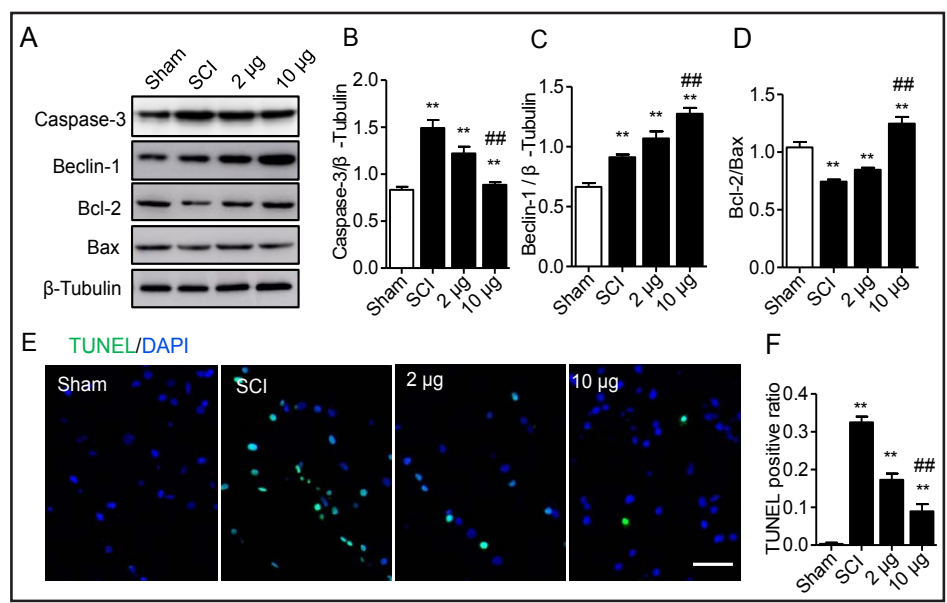

Fig. 5. Exendin-4 inhibits apoptosis. (A-D) Western blot analysis of caspase-3, beclin-1, Bcl-2, Bax, and $\beta$-tubulin. (E) Immunofluorescence assessment of TUNEL. Scale bar $=100 \mu \mathrm{m}$. (F) Proportion of apoptosispositive cells. Values expressed as the mean $\pm \mathrm{SD}$. ${ }^{* *} \mathrm{P}<0.01$, compared with the sham group; ${ }^{\# \#} \mathrm{P}<0.05$, compared with the SCI group. 
dark with a great deal of vacuolization. Compared with the SCI group, the exendin-4 group showed a marked reduction in these pathological changes and a higher number of normal neurons. Most neurons were multipolar with clear karyosomes, with few vacuolar changes (Fig. 6A). Statistical analysis showed that the exendin-4 treatment group had a significantly reduced cavity space $(P<0.05)$ (Fig. 6C). At 4 weeks after surgery, all of the pathological changes were markedly reduced compared with 3 days after the operation, and only a small number of vacuoles and scar formation were seen in the SCI group (Fig. 6B). Statistical analysis revealed a significant difference in the cavity space between the SCI group and sham group $(P<$ 0.05) (Fig. 6C).

Exendin-4 improves rat motor function

We assessed the BBB scores at $1,3,7,14$, and 28 days after SCI, and the BBB scores of the SCI group were significantly decreased

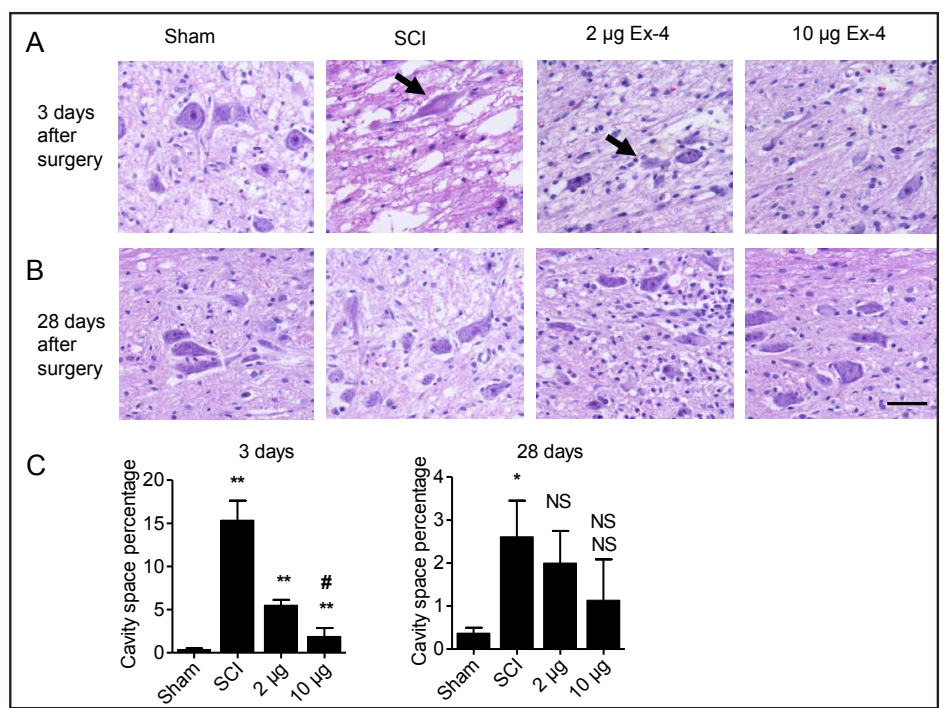

Fig. 6. SERCA2 expression affects histomorphology in rats with SCI. (A) At 3 days after surgery. (B) At 4 weeks after surgery. (C) Statistical analysis of the cavity space. Arrows: apoptotic nerve cell. Hematoxylineosin staining, scale bar $=50 \mu \mathrm{m}$.

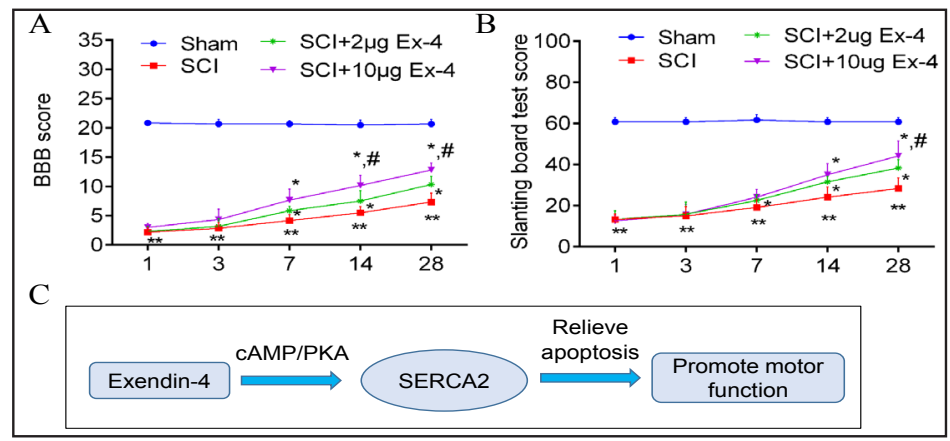

Fig. 7. Exendin- 4 improves motor function in SCI rats. (A) BBB scores at $1,3,7,14$, and 28 days. (B) Slanting board test scores at 1, 3, 7, 14 , and 28 days. ${ }^{*} \mathrm{P}<0.05,{ }^{* *} \mathrm{P}<0.01$, compared with the sham group; ${ }^{\#} \mathrm{P}<0.05$, compared with the sham group. (C) Schematic mechanism of the role of SERCA2 in SCI. compared with those of the sham group $(P<0.01)$. Compared with the SCI group scores, the $2 \mu \mathrm{g}$ and $10 \mu \mathrm{g}$ exendin-4 group scores were significantly increased at 7,14 , and 28 days $(P<0.05)$. Furthermore, the $10 \mu \mathrm{g}$ exendin- 4 group scores were significantly increased compared with the $2 \mu \mathrm{g}$ exendin- 4 group scores at 14 and 28 days $(P<0.05)$ (Fig. 7A).

The slanting board test was performed at $1,3,7,14$, and 28 days after SCI. The slanting board test scores in the SCI group were significantly decreased compared with those in the sham group $(P<0.01)$. Compared with the SCI group scores, the $2 \mu$ g exendin- 4 group scores were significantly increased at 14 and 28 days $(P<0.05)$. However, the $10 \mu$ g exendin- 4 group scores were significantly increased at 7,14 , and 28 days $(P<0.05)$. Furthermore, compared with the $2 \mu$ g exendin-4 group scores, the $10 \mu \mathrm{g}$ exendin-4 group scores were significantly increased at 28 days $(P<0.05)$ (Fig. 7B).

A schematic of the underlying mechanism of the role of SERCA2 in SCI is shown (Fig. 7C). These findings support the neuroprotective effect of exendin- 4 and demonstrate that a high dose of exendin-4 was more effective than a low dose. 


\section{Discussion}

SCI is a fatal condition that usually results in the loss of sensation and movement. Although adequate surgical decompression is the preferred treatment for SCI and results in favorable outcomes, SCI patients experience unpredictable and disastrous postoperative complications $[25,26]$. A series of studies have indicated that SERCA2 has significant protective effects on the heart, liver, and other tissues by reducing endoplasmic reticulum stress and apoptosis [27-30]. Whether SERCA2 has the same effect in the spinal cord is worth exploring.

To investigate the relationship between SERCA2 and apoptosis in the spinal cord, we used a CE intrathecal injection to block SERCA2 in the spinal cord of SCI rats. The results showed that SERCA2 inhibition led to a decrease of Bcl-2 expression and increase of caspase-3 expression. Tymianski and Tator demonstrated that SERCA2 expression abnormalities lead to intracellular calcium homeostasis and the activation of apoptotic caspase cascades [9]. Dremina et al. suggested that the direct interaction of Bcl-2 with SERCA may be involved in the regulation of apoptotic processes in vivo through the modulation of the cytoplasmic and/or endoplasmic reticulum calcium levels required for the execution of apoptosis [31]. Subsequently, we injected cAMP and PKA inhibitors into the medullary sheath of normal rats by intrathecal administration, which demonstrated that the cAMP/PKA signaling pathway can regulate SERCA2 expression. These findings suggest that promoting the expression of SERCA2 can occur through the cAMP/PKA pathway in the spinal cord. At the same time, SERCA2 can promote Bcl-2 expression, inhibit caspase-3 expression, and inhibit apoptosis, which are consistent with the findings of previous studies [9, 32, 33].

The findings from our study also clearly showed that the increased expression of SERCA2 could alleviate apoptosis in SCI rats, which was consistent with its effects in other organs and with the above analysis. In our study, the level of SERCA2 expression was significantly elevated in the exendin-4 group rats and resulted in a marked improvement of motor function. The motor function scores were evaluated by comparison with the SCI group at 7,14 , and 28 days after surgery. In addition, the pathological changes were significantly reduced in the exendin-4 groups, and the number of normal neurons was significantly higher in the exendin-4 groups than in the SCI group. Most of the neurons showed normal nuclei and fewer vacuolar changes. Although the underlying mechanisms involved in SERCA2 protection against heart failure are unknown, previous studies have shown that SERCA2 inhibits endoplasmic reticulum stress, prevents the accumulation of peroxides, and inhibits apoptosis [9, 34, 35]. The present study focused on the relationship between SERCA2 and apoptosis during SCI.

Inhibition of apoptosis could reduce the extent of injury and preserve neurological function, which is closely associated with the accumulation of reactive oxygen species during SCI [36, 37]. Other studies have also shown that the increased expression of SERCA2 effectively reduces cardiomyocyte apoptosis [38]. However, it has yet to be determined whether SERCA2 exhibits neuroprotective effects by reducing apoptosis in SCI. The results from the present study clearly showed that increased SERCA2 expression stimulated by exendin-4 resulted in decreased SCI-induced apoptosis.

Our research has several limitations that deserve special attention. First, although our study suggests that SERCA2 inhibits apoptosis after SCI, further in vitro studies and clinical trials are needed. Second, exendin-4 promotes the expression of SERCA2 and may affect SCI by other pathways, and a SERCA2-specific activator is required for further characterization. Third, this study explored the relationship between SERCA2 and SCI, and further research is needed to determine the mechanism of the effect of SERCA2 on SCI. Future studies should clarify further the protective mechanisms of SERCA2-mediated molecules.

In summary, the neurological effects of exendin-4 on SCI occurred through a mechanism involving, at least in part, SERCA2 activation, which effectively inhibited apoptosis. Overall, we suggest that SERCA2 plays an important role in SCI through apoptosis regulation and 


\begin{tabular}{|c|c|c|}
\hline Cellular Physiology & Cell Physiol Biochem 2018;4 & 17:617-629 \\
\hline and Biochemistry & $\begin{array}{l}\text { Dol: 10.1159/000490017 } \\
\text { Published online: May 25, } 2018\end{array}$ & $\begin{array}{l}\text { O } 2018 \text { The Author(s). Published by S. Karger AG, Basel } \\
\text { www.karger.colom/cpb }\end{array}$ \\
\hline
\end{tabular}

subsequent recovery of histological changes and motor function (Fig. 7C). The results from this study could provide a novel therapeutic target for treating SCI.

\section{Acknowledgements}

This work was supported by the National Key Research and Development Plan of China (2016YFC1101500), the National Natural Science Foundation of China (11672332, 11102235, 31200809, and 81772018), the Key Science and Technology Support Foundation of Tianjin City (17YFZCSY00620), and the National Natural Science Foundation of Tianjin (17JCZDJC35400).

\section{Disclosure Statement}

The authors declare that there are no conflicts of interest.

\section{References}

1 Tator CH, Hashimoto R, Raich A, Norvell D, Fehlings MG, Harrop JS, Guest J, Aarabi B, Grossman RG: Translational potential of preclinical trials of neuroprotection through pharmacotherapy for spinal cord injury. J Neurosurg Spine 2012;17:157-229.

2 Cao Y, Chen Y, Devivo MJ: Lifetime Direct Costs After Spinal Cord Injury. Topics in Spinal Cord Injury Rehabilitation 2011;16:10-16.

-3 Bi Y, Zhu Y, Zhang M, Zhang K, Hua X, Fang Z, Zhou J, Dai W, Cui Y, Li J, You T: Effect of Shikonin on Spinal Cord Injury in Rats Via Regulation of HMGB1/TLR4/NF-kB Signaling Pathway. Cell Physiol Biochem 2017;43:481-491.

- Zendedel A, Mönnink F, Hassanzadeh G, Zaminy A, Ansar MM, Habib P, Slowik A, Kipp M, Beyer C: Estrogen Attenuates Local Inflammasome Expression and Activation after Spinal Cord Injury. Mol Neurobiol 2018;55:1364-1375.

-5 Varma AK, Das A, Wallace G 4th, Barry J, Vertegel AA, Ray SK, Banik NL: Spinal cord injury: a review of current therapy, future treatments, and basic science frontiers. Neurochemical Res 2013;38:895-905.

6 Lopachin R M, Lehning E J: Mechanism of calcium entry during axon injury and degeneration. Toxicol Appl Pharmacol 1997; 143:233.

7 Wolf JA, Stys PK, Lusardi T, Meaney D, Smith DH: Traumatic axonal injury induces calcium influx modulated by tetrodotoxin-sensitive sodium channels. J Neurosci 2001;21:1923-1930.

-8 Kapoor R, Davies M, Blaker PA, Hall SM, Smith KJ: Blockers of sodium and calcium entry protect axons from nitric oxide-mediated degeneration. Ann Neuro 2003;53:174-180.

-9 Kurnellas MP, Nicot A, Shull GE, Elkabes S: Plasma membrane calcium ATPase deficiency causes neuronal pathology in the spinal cord: a potential mechanism for neurodegeneration in multiple sclerosis and spinal cord injury. FASEB J 2005;19:298-300.

$\checkmark 10$ Tymianski M, Tator C H: Normal and abnormal calcium homeostasis in neurons: a basis for the pathophysiology of traumatic and ischemic central nervous system injury. Neurosurgery 1996;38:11761195.

11 Guo H, Cao A, Chu S, Wang Y, Zang Y, Mao X, Wang H, Wang Y, Liu C, Zhang X, Peng W: Astragaloside IV Attenuates Podocyte Apoptosis Mediated by Endoplasmic Reticulum Stress through Upregulating Sarco/ Endoplasmic Reticulum Ca2+-ATPase 2 Expression in Diabetic Nephropathy. Front Pharmacol 2016;7:500.

12 Gong HB, Wang L, Lv Q Wang J: Improved systolic function of rat cardiocytes during heart failure by overexpression of SERCA2a. Eur Rev Med Pharmacol Sci. 2016;20(8):1590-1596.

13 Younce CW, Burmeister MA, Ayala JE: Exendin-4 attenuates high glucose-induced cardiomyocyte apoptosis via inhibition of endoplasmic reticulum stress and activation of SERCA2a. Am J Physiol Cell Physiol 2013;304:C508-518. 


\section{Cellular Physiology Cell Physiol Biochem 2018;47:617-629

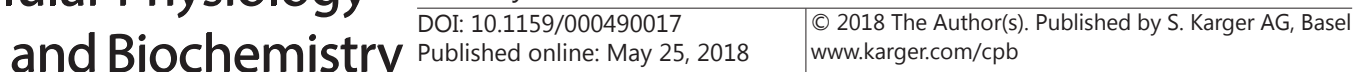

Sun et al.: Exendin-4 Protect Spinal Cord Injury by SERCA2

14 Liang C P, Han S, Gang L, Tall AR: Impaired MEK Signaling and SERCA Expression Promote ER Stress and Apoptosis in Insulin-Resistant Macrophages and Are Reversed by Exenatide Treatment. Diabetes 2012;61:2609-2620.

15 Evan R Zynda, Vitaliy Matveev, Michael Makhanov, Alexander Chenchik, and Eugene S Kandel: Protein kinase A type II- $\alpha$ regulatory subunit regulates the response of prostate cancer cells to taxane treatment. Cell Cycle 2014;13:3292-3301.

-16 Botfield HF, Uldall MS, Westgate CSJ, Mitchell JL, Hagen SM, Gonzalez AM, Hodson DJ, Jensen RH, Sinclair AJ: A glucagon-like peptide-1 receptor agonist reduces intracranial pressure in a rat model of hydrocephalus. Sci Transl Med 2017;9.

17 Basso DM, Beattie MS, Bresnahan JC: A sensitive and reliable locomotor rating scale for open field testing in rats. J Neurotrauma 1995;12:1-21.

18 Dalton GD, Smith FL, Smith PA, Dewey WL: Alterations in brain Protein Kinase A activity and reversal of morphine tolerance by two fragments of native Protein Kinase A inhibitor peptide (PKI). Neuropharmacology 2005;48:648-657.

19 De Wit RJ, Hekstra D, Jastorff B, Stec WJ, Baraniak J, Van Driel R, Van Haastert PJ: Inhibitory action of certain cyclophosphate derivatives of cAMP on cAMP-dependent protein kinases. Eur J Biochem 1984;142:255260.

20 Van Haastert PJ, Van Driel R, Jastorff B, Baraniak J, Stec WJ, De Wit RJ: Competitive cAMP antagonists for cAMP-receptor proteins. J Biol Chem 1984;259:10020-100204.

-21 Suzuki-Mizushima Y, Gohda E, Okamura T, Kanasaki K, Yamamoto I: Enhancement of NGF- and cholera toxin-induced neurite outgrowth by butyrate in PC12 cells. Brain Res 2002;951:209-17.

$22 \mathrm{Hu}$ JZ, Long H, Wu TD, Zhou Y, Lu HB: The effect of estrogen-related receptor $\alpha$ on the regulation of angiogenesis after spinal cord injury. Neuroscience 2015;290:570.

-23 Basso D M, Beattie M S, Bresnahan J C. A sensitive and reliable locomotor rating scale for open field testing in rats. J Neurotrauma 1995;12:1-21.

-24 Rivlin AS, Tator CH: Objective clinical assessment of motor function after experimental spinal cord injury in the rat. J Neurosurg 1977;47:577-581.

25 Seo JY, Kim YH, Kim JW, Kim SI, Ha KY: Effects of Therapeutic Hypothermia on Apoptosis and Autophagy following Spinal Cord Injury in Rats. Spine DOI: 10.1097/BRS.0000000000000845.

26 Yang T, Wu L, Wang H, Fang J, Yao N, Xu Y: Inflammation Level after Decompression Surgery for a Rat Model of Chronic Severe Spinal Cord Compression and Effects on Ischemia-Reperfusion Injury. Neurol Med Chir(Tokyo) 2015;55:578-586.

27 Prasad V, Lorenz JN, Lasko VM, Nieman ML, Huang W, Wang Y, Wieczorek DW, Shull GE: SERCA2 Haploinsufficiency in a Mouse Model of Darier Disease Causes a Selective Predisposition to Heart Failure. Biomed Res Int 2015;2015:251598.

-28 Fajardo VA, Bombardier E, Vigna C, Devji T, Bloemberg D, Gamu D, Gramolini AO, Quadrilatero J, Tupling AR: Co-Expression of SERCA Isoforms, Phospholamban and Sarcolipin in Human Skeletal Muscle Fibers. Plos One 2013;8:e84304.

29 Benali-Furet NL, Chami M, Houel L, De Giorgi F, Vernejoul F, Lagorce D, Buscail L, Bartenschlager R, Ichas F, Rizzuto R, Paterlini-Bréchot P: Hepatitis $C$ virus core triggers apoptosis in liver cells by inducing ER stress and ER calcium depletion. Oncogene 2005;24:4921.

30 Guo H, Wang Y, Zhang X, Zang Y, Zhang Y, Wang L, Wang H, Wang Y, Cao A, Peng W: Astragaloside IV protects against podocyte injury via SERCA2-dependent ER stress reduction and AMPK $\alpha$-regulated autophagy induction in streptozotocin-induced diabetic nephropathy. Sci Rep 2017;7:6852.

31 Dremina ES, Sharov VS, Kumar K, Zaidi A, Michaelis EK, Schöneich C: Anti-apoptotic protein Bcl-2 interacts with and destabilizes the sarcoplasmic/endoplasmic reticulum Ca2+-ATPase (SERCA). Biochem J 2004;383:361-370.

32 Pinton P, Rizzuto R: Bcl-2 and Ca2+ homeostasis in the endoplasmic reticulum. Cell Death Differ 2006;13:1409-1418.

-33 Rodriguez D, Rojas-Rivera D, Hetz C: Integrating stress signals at the endoplasmic reticulum: The BCL-2 protein family rheostat. Biochim Biophys Acta 2011;1813:564-574. 


\section{Cellular Physiology Cell Physiol Biochem 2018;47:617-629 \begin{tabular}{l|l|l} 
DOI: 10.1159/000490017 & O 2018 The Author(s). Published by S. Karger AG, Basel \\
www.karger.com/cpb
\end{tabular} \\ Sun et al.: Exendin-4 Protect Spinal Cord Injury by SERCA2}

-34 Shintani Y, Drexler HC, Kioka H, Terracciano CM, Coppen SR, Imamura H, Akao M, Nakai J, Wheeler AP, Higo S, Nakayama H, Takashima S, Yashiro K, Suzuki K: Toll-like receptor 9 protects non-immune cells from stress by modulating mitochondrial ATP synthesis through the inhibition of SERCA2. EMBO Rep 2014;15:438-445.

-35 Huang H, Joseph LC, Gurin MI, Thorp EB, Morrow JP: Extracellular signal-regulated kinase activation during cardiac hypertrophy reduces sarcoplasmic/endoplasmic reticulum calcium ATPase 2 (SERCA2) transcription. J Mol Cell Cardiol 2014;75:58-63.

-36 Huang F, Li Y N, Yin F, Wu YT, Zhao DX, Li Y, Zhang YF, Zhu QS: Ginsenoside Rb1 inhibits neuronal apoptosis and damage, enhances spinal aquaporin 4 expression and improves neurological deficits in rats with spinal cord ischemia-reperfusion injury. Mol Med Rep 2015;11:3565-3572.

-37 Yu F, Kamada H, Niizuma K, Endo H, Chan PH: Induction of mmp-9 expression and endothelial injury by oxidative stress after spinal cord injury. J Neurotrauma 2008;25:184-195.

38 Qin F, Siwik DA, Pimentel DR, Morgan RJ, Biolo A, Tu VH, Kang YJ, Cohen RA, Colucci WS: Cytosolic H2O2 mediates hypertrophy, apoptosis, and decreased SERCA activity in mice with chronic hemodynamic overload. Am J Physiol Heart Circ Physiol 2014;306:H1453-1463. 